\title{
Convenciones sociales y certificación de los productos pesqueros
}

\section{Social conventions and certification of fishery products}

\author{
Fernando González Laxe ${ }^{1 *}$
}

\begin{abstract}
RESUMEN
Los pescadores siguen tres lógicas. La que está definida por la maximización del beneficio; la relacionada con la abundancia y la situación de los stocks; y la vinculada a las expectativas sociales y económicas. Piensan en "comportamientos ideales"; y, sobre esa base, actúan en función de sus habilidades y con prevalencia de una actitud individual más que colectiva. No obstante, en la medida que los problemas de la gestión pesquera tienen su origen en el análisis de los recursos y en la configuración de los mercados, los pescadores acuden a las convenciones sociales para dar coherencia a sus acciones y poner el acento en la coordinación de los agentes. Metodológicamente, se analizan aquellas convenciones sociales que mantienen una regularidad estable en lo concerniente al proceder de los pescadores y que permiten crear una conducta recurrente en términos de interacciones sociales. El objetivo del trabajo es recoger aquellas prácticas, costumbres, intereses comunes y normas que definen las reglas de comportamiento. Destacamos seis tipos de convenciones sociales: a) las organizativas (que reflejan la actitud de voluntariedad); b) las representativas (que son instrumentos de negociación); c) las reivindicativas (que plasman exigencias ante problemas); d) las comerciales (que refuerzan ventajas diferenciales); e) los procesos de certificación (que subrayan ventajas comparativas y barreras de entradas); y f) las de defensa de la sostenibilidad y bienes comunes (que enfatizan más sobre la preservación que sobre la producción). Finalmente, subrayamos que los productos certificados se han convertido en herramientas políticas y de negociación comercial.
\end{abstract}

Palabras clave: Conducta del pescador, convención social, gestión pesquera, productos certificados y reglas de comportamiento

\begin{abstract}
Fishermen's logic is related to three aspects: profit maximization; abundance and stock situation; and social and economic expectations. They think in terms of "ideal behaviors" and, based on that, they act according to their abilities mostly with an individual rather than a collective attitude. However, when problems in fishery management stem from the analysis of resources and the configuration of markets, fishermen resort to social conventions to give coherence to their actions and emphasize agents' coordination. Methodologically speaking, the paper analyzes
\end{abstract}

1 Catedrático de Economía Aplicada y Director del Instituto de Estudios Marítimos. Facultad de Economía y Empresa. Universidade da Coruña (España). f.laxe@udc.es* ORCID: https://orcid.org/0000-0001-5399-3446 
the social conventions that maintain a stable regularity in fisherman behavior and that allow establishing a recurrent behavior in terms of social interactions. The purpose of the article is to collect those practices, habits, common interests, and norms that define behavior rules. Six types of social conventions are highlighted here: a) organizational (which reflect voluntary attitudes); b) representative (used as negotiation instruments); c) reclaiming (make demands when there are problems); d) commercial (reinforce differential advantages); e) certification processes (underline competitive advantage and entry barriers); and f) social conventions to defend sustainability and common goods (more emphasis on presentation rather than production). Finally, it should be noted that certified products have become political and trade negotiation tools.

Keywords: Fisherman's behavior, social convention, fishery management, certified products, and behavior rules

\section{INTRODUCCIÓN}

Cambios y transformaciones en el sistema mar-industria

El mercado alimentario muestra unas notables transformaciones a lo largo de las últimas décadas. El conjunto de dinámicas relacionadas con las mayores ofertas de productos; los cambios en la demanda; las proyecciones demográficas; la nueva competencia entre productos; la mayor rivalidad entre países; y, sobre todo, las nuevas inquietudes y criterios vinculadas a las decisiones de compra, ya sea en lo que hace referencia a los hogares o en el sector de la distribución y consumo, aceleran dichos cambios. La evolución del sistema mar-industria pasa por transformaciones muy notables. De una parte, las actividades artesanales, tan abundantes en las comunidades costeras, afrontan situaciones de elevada explotación de los recursos, desvalorización de los precios de productos pesqueros y cada vez más una falta de mano de obra (González Laxe, 2019a). De otra parte, la industria pesquera se internacionaliza y se vincula a corporaciones alimentarias de alcance mundial, con una orientación extractiva dirigida a mercados altamente especializados (Österblom et al. 2015) Dichas dinámicas subrayan el aumento de la especialización flexible de la producción, el fraccionamiento de los procesos productivos y la continua adaptación y restructuración de las pesquerías nacionales. Son las respuestas a la creciente demanda de inputs por parte de las corporaciones trasnacionales, para poder abastecer a sus redes de producción y distribución.

Los problemas derivados de la gestión pesquera se han centrado en los aspectos relacionados con los derechos de propiedad de determinados recursos y su incidencia en las condiciones y dinámicas del mercado. En la medida que los peces constituyen un recurso natural, biológico, móvil, renovable, sustractivo, variable y finito, 
su gestión es muy compleja. Los derechos de propiedad se convierten en un instrumento jurídico-económico que ayuda a establecer la relación entre las personas respecto a los recursos naturales. De cara a la regulación y a la ordenación pesquera se utilizan como una corriente de beneficios para unos que los demás productos deben respetar (Omstrom, 1999). Se subrayan cuatro tipos de derechos: a) los derechos de uso (aquellos que conceden el acceso como la captura); b) el derecho de manejo (aquel que regula la participación en la ordenación pesquera mediante el comanejo); c) el derecho de exclusión (que determina quienes poseen la condición de acceso y de captura); y d) el derecho de transferencia (las consideraciones relativas a los derechos de venta, transmisión o legado). Por eso, se afirma que los derechos de propiedad son mecanismos institucionales a través de los cuales los pescadores o comunidades de pescadores tienen el derecho de acceder a un área y a una captura; siendo posible y aplicable su uso individual o colectivo, pudiendo establecer limitaciones de entrada.

La intensa competencia mundial, antaño delimitada por el volumen y el precio, se transforma progresivamente en una nueva dinámica definida por la supremacía de los grandes distribuidores, al amparo de los procesos de liberalización comercial (OECD, 2010). Dichos procesos suponen la consolidación de cadenas más extensas de producción y de distribución que garantizan una mayor disponibilidad de alimentos; la multiplicación de proveedores; y el establecimiento de estándares de calidad. Esto es, el actual sistema mar-industria se encuentra caracterizado por la reorganización de los procesos de producción, distribución y consumo; así como por la presencia de un consumidor más proactivo y atento a los niveles de calidad, aspectos que pueden llegar a ser interpretados como una barrera de entrada para aquellos alimentos a los que se les exige unos estándares impuestos por las recomendaciones y consejos que dominan los mercados de adscripción (Hernández \& Vilaseñor, 2014).

Estas dinámicas permiten advertir cuatro rasgos destacados: a) la necesidad de firmar tratados comerciales entre los países productores y comercializadores; b) un mayor protagonismo de las grandes corporaciones internacionales; c) la existencia de agencias gubernamentales encargadas de la regulación de la producción, distribución e inspección; y d) la existencia de nuevos nichos de mercados que se diferencian y que buscan la especialización al ofertar productos con garantías de calidad y seguridad a los consumidores. Esta visión de las apuestas pesqueras remarca unos determinados ejes que contribuyen a subrayar directrices tendentes hacia una oferta más homogeneizadora, nuevas 
posiciones del consumo doméstico frente a la globalización; una revalorización de los productos procedentes de modelos alternativos e incorporados a las especificidades de un territorio y de un ecosistema; y el advenimiento de una industria innovadora frente a los modelos de producción artesanal (McMichael, 2005).

El trabajo se centra en explicar, en primera instancia, en el funcionamiento del sector pesquero desde la visión de la teoría de las convenciones; es decir, al evaluar los acuerdos entre actores, en la búsqueda y defensa de sus intereses. El segundo apartado, contempla la controversia entre los aspectos concernientes a la gestión pesquera y los aspectos territoriales, analizados desde la óptica de las relaciones sociales. Más tarde, se enfoca el proceso de valorización de los productos pesqueros y la dinámica de las certificaciones de las pesquerías. En este sentido, se contextualizan las instituciones vinculadas a la certificación de las cadenas de custodia y las acciones vinculadas al etiquetado de los productos pesqueros y a los mecanismos de diferenciación de estos. A continuación, se identifican los elementos claves dentro de la cadena de valor y se finaliza con un capítulo de conclusiones.

\section{MATERIALES Y MÉTODOS}

Se procedió a reflejar las distintas lógicas existentes en un sector pesquero, en constante mutación y reorganización, a la luz de las dinámicas de los cambios tecnológicos, la liberalización comercial y las modificaciones de los hábitos en el consumo. A nuestro juicio, los pescadores siguen, normalmente, tres lógicas. La primera, está definida por la maximización del beneficio. Es decir, se busca que la diferencia entre ingresos y costes sea mínima. Por lo menos satisfactoria, dentro de los umbrales del negocio, o que las ratios obtenidas sean objeto de comparación con las expectativas y necesidades que surgen dentro del entorno social y territorial más próximo, lo que se denomina coste de oportunidad. Los pescadores estiman sus expectativas (ejercen cálculos previos de sus rendimientos de ingresos y gastos) y lo hacen asociados a niveles de producción, al incluir los factores vinculados a la incertidumbre y a la imprevisibilidad. Las variables claves de dicha situación vienen dadas por la relación costes/beneficios; por los sistemas de remuneración, por los lugares de pesca; y por los aspectos socioculturales derivados del entorno y del legado histórico y familiar. La segunda lógica está relacionada con las repercusiones de la crisis biológica. Se 
tiene en consideración la distribución de las poblaciones de peces $\mathrm{y}$, por tanto, se busca y se piensa en "comportamientos ideales"; para, sobre ellos, actuar en función de sus habilidades y niveles de competitividad. De esta forma, sobresale la actitud individual más que el comportamiento conjunto de las flotas. Las variables claves de dichas actuaciones vienen dadas por la definición de espacios, de lugares y de zonas de pesca, por un lado; $y$, por los permisos de acceso a dichos emplazamientos, por el otro. Finalmente, es preciso insistir en que los pescadores no actúan de manera uniforme, sino que cada uno de ellos dispone y utiliza informaciones diferentes, provenientes de su experiencia y de las tradiciones heredadas. La tercera lógica está determinada por los enfoques sociales. Esto es, por los derechos sociales, por los comportamientos familiares y los del ámbito territorial. La variable clave sería la conducta de los pescadores relacionada con la dimensión de la flota. No existen, idénticos comportamientos en la flota artesanal, ni costera, ni industrial. Asimismo, el comportamiento del pescador está muy correlacionado con las expectativas relativas a las condiciones de bienestar, supervivencia, entorno, satisfacción laboral y esperanza de vida. Los aspectos relacionados con la pertenencia geográfica y territorial junto al rol desempeñado por el ámbito social-cultural en el que se inscribe, hace que los pescadores formen parte de una identidad particular, como grupo complejo y dinámico, del que se derivan actuaciones singulares en lo que concierne a las relaciones, procesos y vínculos, ya sean muy estrechos entre sí y entre su entorno De esta forma, se conservan y refuerzan sus tradiciones, valores y percepciones presentes, tanto en sus comunidades pesqueras como en lo que atañe a sus vínculos familiares.

El sector pesquero afronta, pues, varios desafíos: a) sufre la influencia de las relaciones de mercado y de los consumidores; b) reduce su control sobre el uso de los recursos pesqueros, dados los procesos de extensificación de las zonas pesqueras y de la dispersión espacial de las capturas; c) queda muy exigido bajo las prácticas de los circuitos de distribución y de las cadenas globales de suministros, para no perder las cualidades organolépticas del recurso; y d) se mantiene muy subordinado a las decisiones gubernamentales, en la medida que no se han generalizado políticas de cogobernanza o acciones colectivas de acción.

El problema de la gestión pesquera tiene, pues, su origen en dos manifestaciones diferentes: en el análisis de los recursos biológicos y en la configuración de los mercados pesqueros y la fijación de los precios. El recurso no es objeto de una apropiación individual formal. El acceso al mismo es, normalmente, libre; de ahí que se utilice el concepto de recurso común. 
Pero, la apropiación efectiva del recurso solo es posible después de sus capturas. Esta ausencia de control sobre el recurso induce a una competencia continua con los demás productores; es decir, todo lo que uno no captura; puedo ser extraído por los demás. Las capturas efectuadas por un pescador dependen, en consecuencia, de la intensidad de la pesca que se ejerce y de la actividad realizada por lo demás pescadores que explotan el mismo recurso. Esta externalidad económica es una fuente permanente de incertidumbre para el pescador. Asimismo, el recurso está sometido a fuertes oscilaciones temporales y a ciclos de abundancia que condicionan su disponibilidad, porque depende del ecosistema y de las características definidas por el hidroclima.

Respecto al mercado y a los precios se asiste a continuas fluctuaciones, que muestran importantes variaciones al socaire de las diferentes cantidades de oferta o de las modificaciones por parte de las demandas. Son relevantes, en primer término, los factores derivados de las conductas y hábitos de los consumidores; en segundo lugar, los aportes procedentes de las importaciones; en tercer lugar, el papel desempeñado por los circuitos de distribución, al ser más potentes y globales; y finalmente, se debe resaltar la dinámica de liberalización de los mercados internacionales, que contribuyen a reforzar la presión sobre el ejercicio pesquero (OECD, 2010), al incrementar los niveles de incertidumbre sobre los mercados y los precios, siendo estos últimos cada vez más exógenos.

\section{Análisis de la teoría de las conven- ciones en el sistema alimentario}

En la década de los noventa, Boltanski \& Thévenot (1991) recurrieron a las teorías de las convenciones para lograr explicar las relaciones entre los procesos de producción y las cadenas de suministro de alimentos. Dichas teorías buscaban dar sentido de coherencia a los diversos fenómenos existentes en el campo de la alimentación. Las mencionadas teorías ponían el acento en la coordinación de los actores y en su comportamiento. Vinculaban las acciones determinadas, en un momento dado, con los modelos normativos que estaban siendo aceptados (Ponte, 2009). Dichos modelos no constituían valores jerárquicos, como si ocurría en la teoría regulacionista (que se focaliza en la producción de los bienes y servicios, así como en la provisión de los servicios públicos, que velan por la competencia entre productores), sino que solo tenían sentido en escenarios específicos; es decir, vinculados a territorios concretos. Por eso, la teoría de la convención plantea que la justificación social de los agentes y sus acciones pueden tener posicionamientos diversos; $y$, que cada mundo posee una coherencia interna, mediante algunos puentes que se interrelacionan entre sí (Ponte, 2009). 
En atención a este enfoque analítico, tanto las formas de producción, distribución y funcionamiento de los mercados, como la canalización de las preferencias de los consumidores, están sujetas a un conjunto de reglas, normas, convenciones e instituciones. Buscan eliminar y minimizar las incertidumbres entre los actores principales. Hernández \& Villaseñor (2014) afirman que "las convenciones son acuerdos que se toman en torno a un producto cuando el precio no es suficiente para calificarlo". Por su parte, Boltanski \& Thévennot (1991) distinguen varios escenarios en los que se llevan a cabo las transacciones de intercambio o de los flujos de bienes y servicios. Cada uno de ellos posee y está creado a partir de paradigmas particulares y posee incidencia en el diverso comportamiento de los actores, con el fin de facilitar la coordinación entre los mismos. Ello ha llevado a poder delimitar que el consumo de alimentos es la resultante, como sugiere Fonte (2002) de una pluralidad de convenciones (doméstica, comercial, industrial, de opinión y cívica) y de la propia coordinación de las actividades económicas del sistema alimentario en su conjunto. Dentro de estas circunstancias, la calidad del producto puede ser interpretada como el proceso de coordinación entre pescadores, por un lado; y consumidores, por el otro. Siendo en este supuesto analítico, el proceso de certificación aquel mecanismo necesario, aunque no suficiente, para recuperar la confianza de los consumidores.

La convención, en este sentido, llega a ser definida como un conjunto de acuerdos individuales negociados dentro de un conjunto específico, afirma Wilkinson (2010); aunque hay que matizar que tales acuerdos se producen en distintos escenarios. Bajo el concepto de la convención, Hernández \& Villaseñor (2014) significan dos visiones teóricas de la calidad del sistema alimentario. La primera, busca analizar las bases estructurales, vinculadas a las exigencias de calidad e inocuidad, como requisitos indispensables para el correcto funcionamiento del sistema alimentario, en virtud de los acuerdos globales suscritos por los países más importantes. La segunda visión, desde una perspectiva más cercana al individualismo, intenta explicar cómo los grupos y los individuos que participan en el sistema alimentario construyen, negocian y fijan sus posturas respecto a la calidad. A juicio de Espinosa \& Sánchez (2013) dichas estrategias son fácilmente comprensibles dadas las apuestas productivas tan diversas, como la acuicultura orgánica, la recualificación productiva, el mercado callejero de productos frescos, las áreas marinas protegidas, el comercio justo o las cadenas de valor. Por lo que tales estrategias son el resultado de perseguir no solo la maximización del beneficio, sino la pertenencia a un 
contexto territorial, con la existencia de vínculos y relaciones sociales, políticas, culturales y de orden económico, que obliguen a construir y mantener un compromiso permanente y a la vez inestable entre diversas lógicas.

Emerge, bajo esta concepción, un nuevo marco teórico sobre la producción, distribución y consumo, donde la relación no está vinculada a un nexo directo entre cantidad y precio (González-Laxe, 2015); sino que se pueden incorporar nuevos criterios o convenciones, que permitan coadyuvar a condicionar las opciones de los consumidores $\mathrm{y}$, por tanto, puedan afectar a las decisiones de los productores, tales como los nuevos desarrollos de food-service, por ejemplo.

Metodológicamente, las convenciones abarcan varias dimensiones. Se contabilizan las convenciones industriales (referenciadas a los procesos); las comerciales (ensalzan las formas tradicionales y el apoyo a las producciones locales); las públicas (dirigidas hacia la reputación y reconocimiento de marcas, distintos y envases); las cívicas (que buscan el bienestar individual y colectivo); y las ecológicas (que evalúan el impacto ambiental). O sea, las convenciones constituyen "acuerdos que guían la acción, que es el resultado de una percepción compartida del entorno" (Parrot et al. 2002). Dicho de otro modo, "es una forma de acuerdo no explícito"; distinguiéndose de los acuerdos contractuales explícitos y concretos. En suma, se prescribe el comportamiento a adoptar, sin poseer la forma de un reglamento escrito al que nos podamos referir.

Distintas aportaciones han sugerido diferentes hipótesis de análisis. Se admiten dos funciones: la de coordinación y la de cooperación entre los individuos. North (1990) sugería que las convenciones poseen una inscripción social; pero que, al mismo tiempo, están conformados por reglas formales; sobre todo, en ausencia de mecanismos ligados a la reputación; de ahí sus continuos cambios. Por su parte, Boyer \& Orléan (2004), subrayan cuatro tipos de transiciones al pasar de una convención a otra: el hundimiento general; la invasión por coerción o por adopción progresiva; la tradición; o el acuerdo. Existen otras teorías por las que las convenciones pueden emerger y transformarse por mimetismo (Orléan, 1989); o por la aceptación de nuevas informaciones (Bessy, 2006).

Bajo el ámbito de su inclusión en espacios territoriales, Salais \& Stopper (1992) definen las convenciones como un conjunto de prácticas habituales y de reglas tácitas que gobiernan los mecanismos de coordinación entre la producción, la transformación, la comercialización y el consumidor final, que revelan cierto entendimiento sobre la calidad y definen, así, la manera como una denominación de origen busca su diferenciación en el mercado global. Subrayan, por tanto, 
las utilidades de la teoría de las convenciones en el sentido de revelar las posibilidades del agregado de valores que se identifican por una idea homogénea, en base a la calidad existente en un territorio determinado.

La teoría de las convenciones constituye un marco de interpretación de la calidad en el sector de la alimentación. Sus principales defensores, Boltanski \& Thévenot (1991), la entienden como un proceso de construcción social y de negociación entre actores. De esta manera, la calidad funciona como un conjunto de reglas tácitas sobre las que se organizan las actividades; y las convenciones son definidas como prácticas, rutinas y acuerdos informales o institucionalizados que permiten, entre otras cosas, identificar la calidad de los productos. Las convenciones pueden reflejar una estrategia comercial y productiva diferenciada, al estar basadas en un conjunto de valores que son compartidos entre los actores de unas cadenas productivas. Por eso, las convenciones no solo son guía para acciones emprendidas o a emprender; sino que también legitiman aquellas acciones que pueden ser llevadas a compromisos o posiblemente a rechazos (Ponte, 2009). Para ello, cada convención social debe cifrar y enumerar cada aspecto concreto, tanto en lo que se refiere a las condiciones de extracción y control de los recursos como a los ámbitos referidos a la calidad y garantías de estos.
La continua aproximación entre productos y territorios junto al desarrollo de los conceptos de proximidad social, aumentan el nivel de identificación entre territorios y productos; lo que contribuye a añadir un valor agregado al consumidor, al punto que los conceptos de genuinidad y tipicidad son el resultado de la historia, cultura y saber-hacer peculiar de un territorio, al constituir atributos de calidad, asociados a prácticas y comportamientos sociales y culturales; acumulados de generación en generación, admitiéndose formas de consumo propias de una región.

Ahora bien, ¿qué pasa en el momento que los productores no llegan a cumplir con los estándares de calidad aplicados por el mercado? Se presentan distintas manifestaciones. De entrada, las empresas transnacionales intentan desplazarlos y enfrentarlos con una competencia desigual dados los asimétricos recursos económicos, científicos, tecnológicos. La pesca costera y del litoral, por su parte, refuerza los sistemas de producción tradicionales, al permanecer en los mercados de proximidad, y en algunos informales, regulados por mecanismos diferentes a las del sistema alimentario mundial. Se basan en las redes de confianza y de lealtad que se establecen entre productores y consumidores que permiten que cuando un producto no posea etiquetas o certificaciones, ni llegue a cumplir con los requisitos exigidos por 
las regulaciones sanitarias, se mantenga en aquellos niveles que satisfagan el gusto de ciertos consumidores y que se identifiquen con los mismos. Responden, pues, en estos casos, a cuestiones de arraigo y de identidad (Goodman, 2003). Estas últimas tendencias están siendo impulsadas por movimientos sociales de consumidores en defensa de los productores artesanales y de los sistemas de producción locales. Cuando se afianza una apuesta por compromisos con la sostenibilidad, salud, implicaciones éticas, defensa de la biodiversidad y protección de recursos naturales, por ejemplo, les hace ser considerados como un nuevo concepto de calidad alternativa.

¿Qué significan estas circunstancias?, Sin duda alguna, abrir un espacio para el desequilibro de poderes dentro de una cadena de valores. Por un lado, las grandes corporaciones buscan, desde la perspectiva convencional, la renta privada máxima; y las pesquerías artesanales, desde la lógica de su regulación, persiguen la generación de una renta social, al promover un entorno social y económico equilibrado, que posibilita dinámicas positivas para los agentes económicos (cooperación, difusión de innovaciones y gobernanza local).

En los supuestos vinculados a los alimentos de calidad diferenciada, las pesquerías artesanales apuestan por las convenciones domésticas, cívicas y ecológicas en la medida que las dos primeras (domésticas y cívicas) combinan las economías alternativas y de base local como los productos típicos; al revalizar dichos productos y territorios; se evocan valores de pertenencia a tradiciones, historia y cultura: en tanto que las convenciones ecológicas, que hacen hincapié en la conservación y uso responsable de los recursos naturales, tienen más posibilidades de impulsar dinámicas favorables a un territorio, dada la mayor participación de los actores locales en todos los procesos sociales, económicos y políticos (Salas \& Gaertner, 2004; Berkes \& Nayak, 2018; Jentoft et al. 2018; Pauly, 2018; Song et al. 2018).

\section{RESULTADOSYDISCUSIÓN}

El actual sistema pesquero internacional está sometido a constantes transformaciones que tienen como objetivo responder mediante una reorganización de sus unidades operativas a los nuevos procesos de producción y distribución de los productos pesqueros. Asimismo, se contemplan nuevas disposiciones institucionales en lo referente a las calidades, estándares, criterios de salud, seguridad, trazabilidad y competencia de estos. Es por ello, que, para algunos, la calidad alimentaria se está convirtiendo en una auténtica barrera de entrada (Hatamaka \& Busch, 2008); mientras que, para otros, los aspectos de revalorización están 
adscritos a los criterios específicos de un territorio, pues las peculiaridades de su ecosistema permiten incorporar nuevos ingredientes y mantener ciertas condiciones socioculturales ligadas a la historia (Mansfield, 2003; Fonte et al. 2006; McMichael, 2009).

En la actualidad, destacan varios rasgos diferenciales: a) la existencia de grandes corporaciones empresariales, que aprovechan la segmentación de los procesos productivos y la restructuración de las pesquerías nacionales; b) una más intensa competencia, afincada en las estrategias de diferenciación y en la calidad de los productos, más que en los términos de volumen y de los precios de los mismos; c) el mayor poder de los grandes minoristas (supermercados y cadenas de intermediación en lo tocante a las relaciones de mercado; y d) el liderazgo empresarial, basado en el mayor liberalismo comercial y en el fortalecimiento del comercio mundial que influyen en los nuevos patrones y hábitos de consumo. Es decir, estamos en presencia de una inserción, cada vez más intensa, a lo largo de cadenas globales en lo tocante a la producción y a la distribución; a la vez que se asiste a una multiplicación de proveedores, impulsados por las propias corporaciones multinacionales o por el desarrollo de proyectos con menor recorrido mercantil.

Cada vez más, las agencias gubernamentales de regulación son quienes se encargan de verificar la producción, distribución e inspección. Los propios tratados comerciales entre nacionales permiten el afianzamiento de las normas; por eso, en la actualidad, las grandes corporaciones cobran protagonismo, lo que trasciende sus actuaciones (Österblom et al. 2015; Noel, 2013). Las nuevas condiciones de regulación comienzan a definir los nuevos estándares de calidad que, en ocasiones, se convierten en barreras de entrada en el sector. Paralelamente, algunos Estados y Gobiernos regionales definen normas propias con el objetivo de crear nichos de productos propios y abrir nuevos mercados, con el propósito de poder diferenciar y estratificar a los competidores, al afianzar las condiciones de calidad, seguridad alimentaria y eliminación de riesgos.

Si se examina la actual composición del sector se advierte que una parte de la producción artesanal se siente desplazada, debido al resurgir de una industria pesquera manufacturera centrada en productos más procesados, a gran escala, estandarizados, sin un anclaje territorial específico y ofertándose en mercados sin restricciones estacionales respecto a las producciones locales (Fonte, 2002). Estas nuevas tendencias homogeneizadoras permiten difundir diferentes cambios en los hábitos de consumo $\mathrm{y}$, en ciertas circunstancias, alientan distintos paradigmas de la globalización. En ocasiones, hay respuestas por parte de los productores locales que buscan defender 
y revalorizar productos alimentarios alternativos vinculados a ciertos rasgos o valores (como la sostenibilidad o las referencias locales, por ejemplo), frente a los productos originarios de procesos industriales.

La regulación, que había sido hasta hace poco tiempo una facultad exclusiva del Estado, pasa a ser "dirigida" por el sector privado multinacional, a través de una compleja red de certificados que aseguran y garantizan el cumplimiento de ciertos estándares de calidad y seguridad a través de consorcios y agencias. Los sistemas de etiquetado responden a tres principios. El primero, que los sistemas deben estar asociados a procesos extractivos sostenibles; el segundo, que el aumento de la concienciación de la sociedad ha de ser constante y permanente; y el tercero, que los propios sistemas han de ser un aliciente para soportar costes más altos en los procesos de producción, etiquetado y trazabilidad. La FAO (2009) se centró tanto en las certificaciones de las pesquerías como en las cadenas de custodia. Las certificaciones orientadas a las empresas armonizan estándares y con ello se trasmite a los consumidores que los productos cumplen con los niveles establecidos de calidad, seguridad y que se haya extraído y elaborado teniendo en cuenta los criterios de sustentabilidad, higiene, reglas medio-ambientales y condiciones de trabajo. En consecuencia, las certificaciones se han convertido en el eje regulador del nuevo régimen alimentario, al imponer a los agentes económicos determinadas concepciones de calidad e inocuidad, que se aplican a lo largo de toda la cadena de proveedores tal y como se exponen en los trabajos de Zepeda et al. (2019); y González-Laxe (2019b). En suma, se trata de homogenizar una oferta dispersa; de evitar riesgos asociados con la contaminación y las enfermedades. De ahí que se yuxtapongan dos objetivos: los nuevos requisitos de las empresas y los niveles de calidad de los productos, características ambas que constituyen los ejes de respuesta a las nuevas necesidades y exigencias de los consumidores. No hay, pues, ninguna duda que esta forma de regulación/ adaptación evidencia asimetrías entre los distintos países, a la vez que permiten visualizar nuevas realidades de poder dentro de las cadenas del valor de la industria pesquera (Kaplinsky, 2000; González-Laxe et al. 2018). Distinguimos, entonces, dos lógicas: la lógica de la producción y la lógica de la regulación. La primera afecta a las relaciones inter-individuales internas, en una cultura vinculada a un mundo local. La segunda, busca su inserción en un mercado más amplio, en donde predominan las exigencias de transparencia y de una mayor competencia.

Bajo este contexto, la teoría de las convenciones aplicada a las pesquerías pone el acento en la movilización de los actores, en su comportamiento 
y en sus influencias. Trata de desarrollar acciones que posean y adquieran el valor de unas normas que sean aceptadas por la cuasi-totalidad de los agentes. Dichos modelos de análisis no son consideraciones jerárquicas, ni son válidos para todas las sociedades. Tampoco son inevitables. Tienen, exclusivamente, sentido en escenarios específicos; es decir, en un tiempo determinado y en una localización concreta. De esta manera, las convenciones pueden justificar acciones en diferentes situaciones y localidades, pues generan interpretaciones capaces de interrelacionar efectos y variables. Por eso, son defendidas como afirma Hernández \& Villaseñor (2014) como "acuerdos que se toman en torno a un producto cuando el precio no es suficiente para calificarlo". Para, de esta forma, justificar el poder operar y sus resultados en múltiples acciones, como manifiesta Ponte (2009).

El proceso de valorización de los productos pesqueros se ha convertido, por consiguiente, en uno de los ejes principales del debate. Las herramientas de cara a lograr una mayor efectividad del proceso se centran, desde hace una década, en las acciones de certificación y eco-etiquetado de los productos del mar. Las certificaciones de estos son el procedimiento por el que un tercero concede una garantía escrita o equivalente de que una pesquería se ajusta a ciertas normas y de que exista una cadena de custodia que garantiza un procedimiento que posibilita una diferenciación respecto a otros productos y que concede credibilidad a la propia pesquería. Es, por tanto, un distintivo.

A pesar de que la certificación y los sistemas de rastreabilidad son instrumentos consolidados, que se utilizan para verificar la integridad de las cadenas de suministros de los productos, no todos los sistemas tienen porqué poder fusionarse. Existen diferentes sistemas que cubren distintas fases y procesos. Asimismo, los sistemas de certificación pueden ser normales, contractuales o voluntarios, lo que suscita una cierta incertidumbre a la hora de definir y evaluar sus consecuencias.

Las etiquetas son identificaciones que reflejan información, alojando aquellos datos referentes a los lugares de captura, trazabilidad y calidad. Cada vez es mayor la cantidad de pesquerías y de producciones acuícolas que están siendo certificadas. En 2015, la proporción de pesca certificada sobre la producción global ascendió al 14.2\%, lo que muestra asimismo una tendencia creciente en los últimos ocho años (Potts \& Haward, 2016). Varias características subrayan a las iniciativas encargadas de la certificación pesquera. La mayor parte de estas pertenecen a la sociedad civil o a un partenariado entre la propia sociedad civil y el sector privado de la actividad. Han sido constituidas en los últimos veinte años. Son mayores los porcentajes de 
certificación en el mundo de la captura que en el de la acuicultura; y predominan las iniciativas en los países en desarrollo más que en los desarrollados (Cuadro 1).

La certificación es una herramienta política y de negociación comercial en la que se desenvuelve la pesquería. Distinguimos dificultades y oportunidades. Entre los inconvenientes: a) el coste de la certificación, que aumenta a medida que se incrementan las mejoras en el manejo; $b$ ) las exigencias rigurosas en las normas a aplicar, debido a la obligatoriedad de implementar en el proceso de certificación los retos de carácter tecnológico, técnico y de investigación; c) la escasez de promoción de las especies certificadas, en la medida que se efectúa y se limita a las propias organizaciones no gubernamentales (ONG's) y al ámbito de los minoristas; d) el desconocimiento de los certificados, tanto por la propia

Cuadro 1. Distribución de las principales especies pesqueras producidas y certificadas

Table 1. Distribution of the main fish species produced and certified

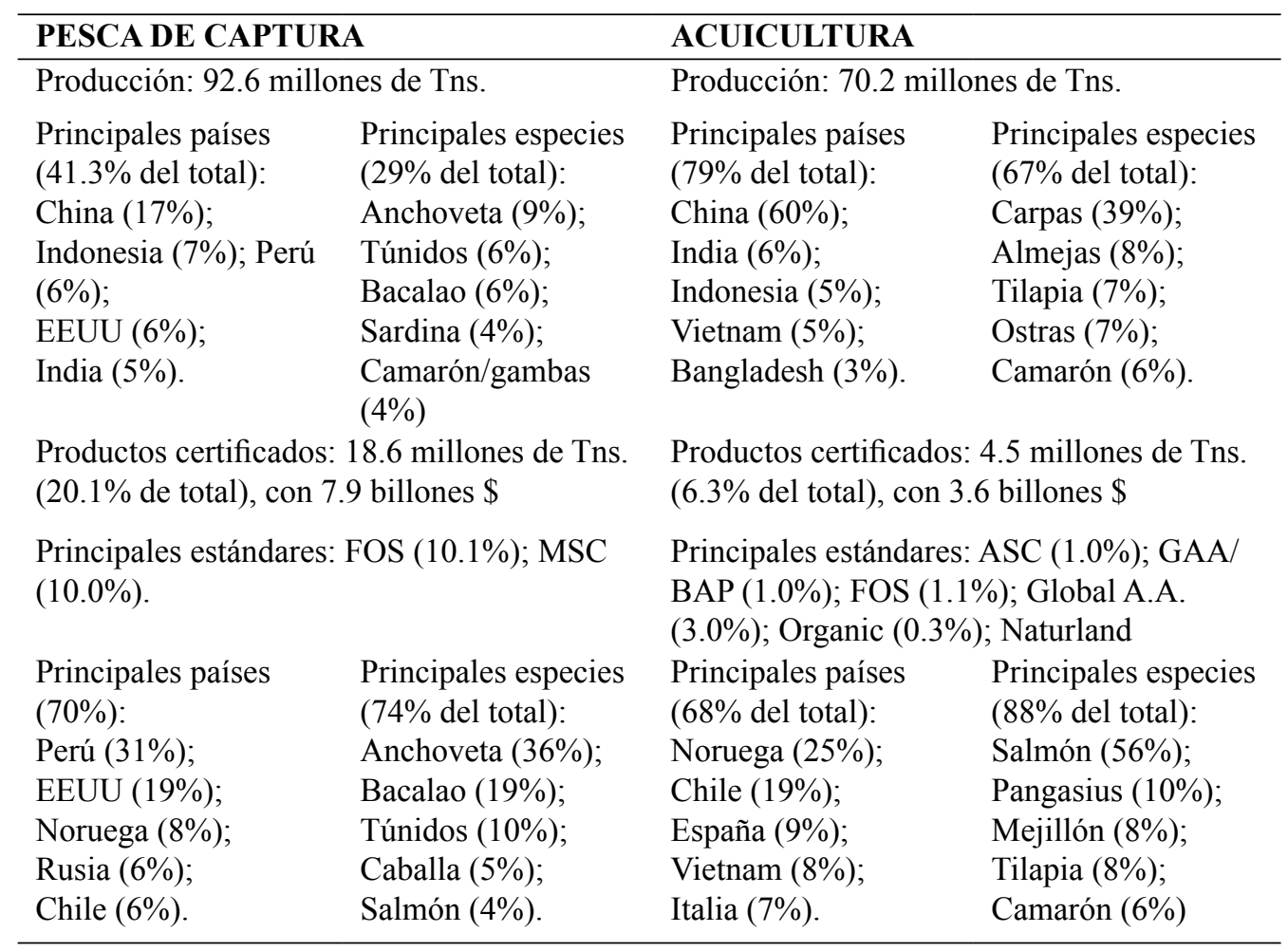

Adaptado de Potts \& Haward (2016). 
industria pesquera como por los Gobiernos; e) el no guardar relación con las demandas de los consumidores. Y, entre las oportunidades: a) una apuesta por la gobernabilidad y sostenibilidad de las pesquerías; b) la aplicación de una metodología de análisis del riesgo que permite y contribuye a garantizar la sostenibilidad; c) la aplicación de mecanismos que coadyuvan a lograr y conseguir una sostenibilidad responsable y una presencia en los mercados internacionales como productos diferenciados; y d) la creación de nuevos nichos de mercado.

El conjunto de los pescadores busca mejorar la comercialización, la información y la seguridad. A medida que el comercio internacional y el consumo de productos pesqueros aumentan, las certificaciones se han ido aplicando de manera más rigurosa. La aceptación de estos criterios facilita la creación de un sobreprecio a los productos con etiqueta sobre los no etiquetados (Song-Grudvag et al. 2013); estableciéndose distintos criterios a la hora de formular acciones de cara a una mayor valorización (Cuadro 2).

A pesar de estas consideraciones, no todo es fácil. Existen varios obstáculos para su implementación. El primero, es que en las regiones y países en desarrollo es mucho más dificultoso, en la medida que la política pesquera ha de contemplar derechos de propiedad sobre los recursos (Kaiser \& Edward-Jones, 2006). El segundo

Cuadro 2. Fundamentos en el procedimiento de valorización de los productos pesqueros de alta calidad

Table 2. Basics of the procedure to assess high-quality fishery products

\begin{tabular}{lll}
\hline Criterio & Dimensión & Efecto \\
\hline Tipicidad del arte & $\begin{array}{l}\text { Define la calidad específica de una } \\
\text { especie a través de las técnicas de } \\
\text { pesca utilizada y del know-how de los } \\
\text { pescadores. }\end{array}$ & $\begin{array}{l}\text { Diferenciación } \\
\text { elevada }\end{array}$ \\
$\begin{array}{l}\text { Reglas de origen de los } \\
\text { productos }\end{array}$ & $\begin{array}{l}\text { Define la calidad intrínseca del caladero o } \\
\text { zona de pesca }\end{array}$ & $\begin{array}{l}\text { Diferenciación } \\
\text { media }\end{array}$ \\
Proceso de producción & $\begin{array}{l}\text { Define la calidad del trabajo, cualificación } \\
\text { y a acreditación de la mano de obra. } \\
\text { Enfatiza sobre los criterios de } \\
\text { funcionamiento y conservación de lo }\end{array}$ & $\begin{array}{l}\text { mediana } \\
\text { recursos. }\end{array}$ \\
& $\begin{array}{l}\text { Define la calidad del producto } \\
\text { seleccionado. } \\
\text { Evalúa las extracciones atendiendo a las } \\
\text { condiciones propias del producto. }\end{array}$ & Estandarización \\
& elevada \\
\hline
\end{tabular}


obstáculo, es que ciertos mercados locales no llegan a ser aptos o idóneos para el interés de los productos certificados, en la medida que en ellos no se obtienen unas economías de escala favorables, ni se concentra una demanda amplia. Y, una tercera dificultad, es que hasta el momento los productos certificados en materia pesquera y acuícola se han orientado hacia especies y mercados ubicados en países desarrollados; subrayando, por tanto, un gap comercial muy notable que observa la procedencia de los recursos. Las experiencias de los consumidores indican, por su parte, que cada vez es mayor la existencia de un elevado porcentaje de consumidores que están dispuestos a comprar pescado eco-etiquetado, catalogado como sustentable; que dicha decisión de compra está influenciada por el conocimiento y las características del producto; que existe intención y aceptación de respuestas éticas en el ejercicio de la pesca, el manejo, la conservación y la presentación; y que, finalmente, los consumidores están dispuestos a pagar un precio premiun por pescado eco-etiquetado (Roheim et al. 2011; Song-Grundvag et al. 2013; Pérez-Ramírez et al. 2015).

No obstante, en ciertos países, EE. UU., Noruega o el Reino Unido, los condicionantes aparecidos son numerosos y se concentran en tres rasgos: a) los sistemas eco-etiquetados están condicionados por las especies y las regiones geográficas; b) están determinados por las características y rasgos de los consumidores; y c) están diferenciados por la reputación de las organizaciones científicas.

De esta manera, los escenarios posibles se delimitan a tres supuestos:

a) Escenario a favor. Cuando las condiciones ideales supongan: obtener certificados en el menor tiempo posible; que existan campañas y acciones de promoción y mejora de la reputación; y, que los costes relacionados con la implementación sean financiables debido a que la inversión sea menor que la ganancia.

a) Escenario actual. Cuando se vuelvan a recalcar las condiciones vigentes; esto es, mayores presiones del consumidor y del propio mercado hacia productos etiquetados y certificados; cuando se mantenga la reputación y una mediana presión de los intermediarios; y cuando no haya sobreprecios; pero sí prestigio.

a) Escenario en contra. Cuando los factores externos sean mínimos $\mathrm{y}$, por lo tanto, los incentivos sean menores a los costes de certificación.

¿Cuáles son, en consecuencia, las claves principales en un contexto de mayor competencia y rivalidad? A modo de síntesis, la presión de los intermediarios (es decir, las cadenas de suministro y distribución); la presión de los grupos de pescadores y de las 
ONG; y el comportamiento de los pre- siendo complementaria de las medicios al consumidor que vienen muy das de gestión. Sin embargo, subsiste condicionados por el conocimiento/ bastante confusión en torno a la aplidesconocimiento del producto y por cación de los diferentes sistemas de el interés/desinterés por adquirir un producto diferenciado. En consecuencia, la certificación pesquera incide de manera muy directa en las políticas de conservación y ordenación pesquera; certificación, ya sea promovido por los organismos de certificación, ya sea derivado de una aplicación nacional o internacional (Christian et al. 2013). (Cuadro 3).

Cuadro 3. Cuadro de indicadores y marcos de actuación de los productores Table 3. Indicators and action framework of producers

\begin{tabular}{|c|c|c|c|c|}
\hline Indicadores & $\begin{array}{l}\text { Marcos de } \\
\text { actuación }\end{array}$ & $\begin{array}{l}\text { Productos a } \\
\text { verificar }\end{array}$ & Ventajas & Inconvenientes \\
\hline $\begin{array}{l}\text { Certificación } \\
\text { de calidad }\end{array}$ & $\begin{array}{l}\text { Actuación } \\
\text { colectiva }\end{array}$ & $\begin{array}{l}\text { Transformados } \\
\text { Acuicultura }\end{array}$ & $\begin{array}{l}\text { Reconocimiento } \\
\text { de nichos de } \\
\text { mercado. Productos } \\
\text { territorializados. }\end{array}$ & $\begin{array}{l}\text { Procedimiento } \\
\text { prolijo. } \\
\text { En ocasiones no } \\
\text { responde a las } \\
\text { demandas. }\end{array}$ \\
\hline $\begin{array}{l}\text { Marca } \\
\text { colectiva }\end{array}$ & $\begin{array}{l}\text { Actuación } \\
\text { colectiva }\end{array}$ & $\begin{array}{l}\text { Pesca. } \\
\text { Transformados } \\
\text { Acuicultura }\end{array}$ & $\begin{array}{l}\text { Marcas globales. } \\
\text { Desarrollo de la } \\
\text { integración en } \\
\text { cadenas. } \\
\text { Productos } \\
\text { territorializados }\end{array}$ & $\begin{array}{l}\text { Posibles } \\
\text { restricciones } \\
\text { sobre el origen } \\
\text { del producto. Sin } \\
\text { reconocimiento } \\
\text { oficial. }\end{array}$ \\
\hline $\begin{array}{l}\text { Marca } \\
\text { independiente }\end{array}$ & $\begin{array}{l}\text { Actuación } \\
\text { individual }\end{array}$ & $\begin{array}{l}\text { Pesca. } \\
\text { Transformados } \\
\text { Acuicultura }\end{array}$ & $\begin{array}{l}\text { Identificación del } \\
\text { productor. } \\
\text { No existe } \\
\text { especificación }\end{array}$ & $\begin{array}{l}\text { Dificultad de } \\
\text { enraizar la marca. } \\
\text { Elevado gasto en } \\
\text { comunicación y } \\
\text { marketing. }\end{array}$ \\
\hline $\begin{array}{l}\text { Etiquetas } \\
\text { ecológicas }\end{array}$ & $\begin{array}{l}\text { Actuación } \\
\text { mixta }\end{array}$ & Pesca & $\begin{array}{l}\text { Mercados singulares } \\
\text { y demandas propias }\end{array}$ & $\begin{array}{l}\text { Diversidad de } \\
\text { etiquetas. Elevado } \\
\text { coste de la } \\
\text { certificación. }\end{array}$ \\
\hline $\begin{array}{l}\text { Acuicultura } \\
\text { ecológica }\end{array}$ & $\begin{array}{l}\text { Actuación } \\
\text { individual }\end{array}$ & Acuicultura & $\begin{array}{l}\text { Mercados singulares } \\
\text { y demandas propias }\end{array}$ & $\begin{array}{l}\text { Complejidad en } \\
\text { el cumplimiento } \\
\text { de las } \\
\text { especificaciones. }\end{array}$ \\
\hline
\end{tabular}




\section{CONCLUSIONES}

El panorama pesquero mundial muestra dinámicas significadamente contradictorias (Le Roux \& Noel, 2007). Por una parte, una atomización relativa de los espacios de producción (dadas las mayores y diferentes condiciones de acceso a los lugares de pesca y a la vigorización de la actividad pesquera en determinados países en desarrollo) y una fuerte concentración de los espacios de consumo en los países occidentales. De otra parte, una intensa mundialización de los flujos comerciales que refuerzan las tendencias asimétricas. Es decir, no existe un sistema-mundo unificado en materia pesquera (Noel, 2013). Dicho en otras palabras, el sistema-mundo pesquero se presenta como un sistema de desigualdades continuas en todos los espacios de análisis en donde los polos de crecimiento acumulan riquezas o niveles de concentración más fuertes que tapan las capas de pobreza y de exclusión de determinadas áreas y territorios.

La globalización pesquera tiende a englobar a todos los espacios y escalas (ya sea superiores o inferiores) en un mismo movimiento de unificación e inserción (a través de los flujos comerciales y por medio de los actores) $\mathrm{y}$ al mismo tiempo trata de uniformizar los modelos de producción y de consumo. Con ello no se puede llegar a afirmar que dicho proceso sea universal, ya que tales dinámicas se inscriben en procesos inestables, contradictorios, de des-estructuración productiva y de estructuración de espacios; lo que permite entrever una dinámica un poco caótica del planeta océano en su concepto de articular espacios, territorios $\mathrm{y}$ redes.

Bajo esta concepción, las tensiones en el seno del sistema pesquero mundial son constantes e intensas; generando situaciones de incertidumbre y de complejidad, derivadas de las interacciones existentes entre las variables (Mahon et al. 2008). La globalización del sistema pesquero subraya la fuerte inter-acción entre el sistema mundo haliéutico con el sistema-tierra, que sirve de soporte espacial. De ahí la necesidad de abrir los debates a las escalas espaciales y temporales, a las nuevas cuestiones relacionadas con la gobernanza y a las acciones tendentes hacia una mejor regulación de las actividades marítimas que incluyan la gestión de los recursos y de los espacios donde reposan estos (Cole, 2003).

Finalmente, en el actual funcionamiento del sector pesquero se conforman seis tipos de convenciones sociales en torno a los productores: a) las organizativas (en función de la asociación de productores) que visualizan los rasgos y la actitud de voluntariedad respecto a la unión de los productores; $\mathrm{y}$ muestran rigidez en cuanto a las normas; b) las representativas (como instrumento de negociación y órgano de consulta) que ayuda a la toma de 
decisiones y definen los criterios de adopción de los agentes, que delimitan tanto los incluidos como los excluidos; c) las reivindicativas, esto es, aquellas que reflejan evaluación, transparencia y exigencias ante los problemas planteados y suscitados en el quehacer cotidiano o estratégico; d) las comerciales, con sus estrategias ofensivas y defensivas, dirigidas a reforzar las ventajas diferenciales y a aminorar las desigualdades de los factores de producción; e) las referidas a los procesos de certificación, que provocan ventajas comparativas en los mercados diferenciales, al limitar las barreras de entrada; y f) las aplicadas a la sostenibilidad y la defensa de los bienes comunes, que buscan la prevalencia de los contenidos basados en la preservación más que los objetivos de producción.

En consecuencia, el proceso de gestión pesquera supone abordar una triangulación entre la administración pública; los profesionales o agentes; y los científicos. Entre los dos primeros existe un flujo relativo de comunicación relativo a las acciones a adoptar. Entre los profesionales y los científicos se contabiliza un flujo de información y de trasmisión de datos. Y, por último, entre los científicos y la administración se localiza el flujo de los consejos. Sin embargo, la mencionada triangulación no siempre es correcta y genera resultados óptimos.

\section{REFERENCIAS}

Berkes, F. \& Nayak, P. K. (2018). Role of communities in fisheries management: "one would first need to imagine it". Maritime Studies, 17, 241-251.

Bessy, C. (2006). La place de l'intersubjectivité et du commun dans l'économie des conventions. En F. Eymard-Duverbay (dir.). L'économie des conventions, Tome I. Débats. Francia. La Découverte.

Boltanski, L. \& Thevenot, L. (1991). De la justification. Les économies de la grandeur. Francia. Ed. Gallimard.

Boyer, R., Orléan, A. (2004). Persistence et changement des conventions. Deux modèles simples et quelques illustrations. En A. Orléan (dir.), Analyse économique des conventions (pp. 243271) Francia. Presses Universitaires de France.

Christian, L., Ainley, D., Baileye, M., Dayton, P., Holevar, J., Levinef, M., ... \& Jacquet, J. (2013). A review of formal objections to MSC Fisheries Certification. Biol. Conserv., 161, 10-17.

Cole, H. (2003). Contemporary challenges: globalisation, global interconnectedness and that are not plenty more fish in the sea. Fisheries, governance and globalisation: is there a relationship. Ocean Coastal Manag., 46(1-2), 77-102.

Espinosa, A. \& Sánchez, J. L. (2013). El mercado español del bacalao en salazón: una aproximación regional desde la geografía de las convenciones. Estudios Geográficos, 74(275), 439-467.

FAO. (2009). Directrices para el etiquetado de pescado y productos pesqueros de la pesca de captura marina. Roma. FAO.

Fonte, M. (2002). Food Systems consumptions models and risk perceptions in 
late moderning. Int. J. Sociol. Agric. Food, 10(1), 13-21.

Fonte, M., Acampora, T. \& Sacco, V. (2006). Desarrollo rural e identidad cultural: reflexiones teóricas y casos empíricos. Chile. Centro Latinoamericano para el Desarrollo Rural.

González-Laxe, F. (2015). Cuestiones biológicas y económicas para explicar ciertas incongruencias de la política pesquera de la Unión Europea. CICIMAR-Oceánides, 30(2), 13-25.

González-Laxe, F. (2019a). Pescadores migrantes: análisis de su dinámica e inclusión social. Rev. Est. Econ. Apl., 37(3). 18-30.

González-Laxe, F. (2019b). Análisis de las controversias respecto al etiquetado. En G. Ponce \& F. González-Laxe. Gobernanza Pesquera (pp.181-213). México. Instituto Politécnico Nacional.

González-Laxe, F., Martin, F., Novo, I., Martin-Palmero, F. (2018). A global industry fishery. Ocean Coastal Manag., 50, 73-82.

Goodman, D. (2003). The quality "turn" and alternative local practices: reflections agenda. J. Rural Stu., 19, 1-17.

Hatamaka, M. \& Bush, L. (2008). Third-Party certification in the global agrifood system: an objective or social mediated governance mechanism. Sociología Ruralis, 48(1), 73-91.

Hernández, Ma . \& Villaseñor, A. (2014). La calidad en el sistema agroalimentario globalizado. R. Mexicana Soci., 76(4), 557-582.

Jentoft, S., Bavinck, M., Alonso-Población, E., Child, A., Diegues, A., Kalikoski, D., ... \& Solís, V. (2018). Working together in small-scale fisheries: harnessing collective action for poverty eradication. Mar. Stu., 17. 1-12.

Johnston, R. I, Wessels, C. R., Donath, H. \& Asche, F. (2001). Measuring Consumer preference for ecolabeled seafood an international comparison. J. Agri. Resour. Econ., 26(1), 20-39.

Kaiser, M. J. \& Edward-Jones, E. (2006). The role of ecolabeling in fisheries management and conservation. Cons. Biol., 20(2), 392-398.

Kaplinsky, R. (2000). Globalization and unequalization: what can be learned from value chain analysis? $J$. Dev. Stud., 37(2), 117-146.

Le Roux, S. \& Noël, J. (2007). Mondialisation et conflits autour des ressources halieutiques. Ecol. Polit., 34, 69-82.

Mahon, R., Mc Conney, P. \& Roy, R.N. (2008). Governing fisheries as complex adaptative systems. Mar. Policy, 32(1), 104-122.

Mansfield, B. (2003). Spatializing globalization: geography of quality in the seafood industry. Econ. Geography, 79, 1-16.

McMichael, P. (2005). Global development and the corporate food regime. New Dir. Sociol. Global Dev., 11, 269-303.

McMichael, P. (2009). A food regimen genealogy. J. Peasant Stu., 36(1), 139-169.

Noel, J. (2013). Le système monde aquatique: panorama géographique d'une filière globalisée. Inform. Géo., 77(1), 76-100.

North, D. C. (1990). Institutions, Institutional Change and Economics Performance. EE. UU. Cambridge University Press.

OECD. Organisation for Economic Co-operation and Development. (2010). Globalisation in fisheries and aquaculture. Opportunities and challenges. Paris. OECD Publishing.

Omstrom, E. (1999). Governing the commons: the evolution of Institutions for Collective Action. EE. UU.: Cambridge University Press.

Orléan, A. (1989). Pour une approche cognitive des conventions, En J. P. Dupuy, 
F. Eymard-Duvernay, O. Favereau, A. Orléan, R. Salais \& L. Thévenot, (Eds.) L'économie des conventions, Revue Économique, 40(12), 341-372.

Österblom, H., Jouffray, J. B., Folke, C., Crona, B., Troell, M., Merrie, A. \& Rockström, J. (2015). Transnational corporations as 'Keystone Actors' in Marine Ecosystems. PLOS One, 10(5). e0127533. https: //doi.irg//10.1371/ journal.pone.0127533

Parrot, N., Wilson, N. \& Murdoch, J. (2002). Spatializing quality: regional protection and the alternative geography of food. Eur. Urban Reg. Stud., 9, 241-261.

Pauly, D. (2018). A vision for marine fisheries in a global blue economy. Marine Policy, 87, 371-374.

Pérez-Ramirez, M., Castrejón, M., Gutiérrez, N. \& Defo, O. (2015). The MSC certification in Latin-America and the Caribbean: a review of experiences potentials and pitfalls. Fis. Res., 182, 50-58.

Pérez-Ramirez, M., Phillips, B., Lluch-Belda, D. \& Lluch-Cota, S. (2012). Perspectives for implementing fisheries certification in developing countries. Mar. Policy, 36, 297-302.

Ponte, S. (2009). Governing through quality: conventions and supply relations in the value chain for South African wine. Sociologia Ruralis, 49(3), 236-257.

Potts, J. \& Haward, M. (2016). State of Sustainability Initiatives review: standards and the Blue Economy. Canadá. IISD/ IIED/FAST.

Roheim, C. A., Asche, F. \& Insignares, J. (2011). The elusive price Premium for ecolabelled products: evidence from seafood in the UK market. J. Agr. Econ., 62, 655-668.
Salais, R. \& Storper, M. (1992). The four worlds of contemporary industry. Cambridge J. Econ., 16,169-193.

Salas, S. \& Gaertner, D. (2004). The behavioural dynamics of fishers: management implications. Fis. Fish, 5, 153-167.

Song-Grudwag, G., Larsen. T. \& Young, J. (2013). The value of line-caught and other attributes: an exploration of price premium for chilled fish in UK supermarkets. Mar. Policy, 38, 41-44.

Song, A., Johnsen, J., Morrrison, T. (2018). Reconstructing governability: How fisheries are made. Fis. Fish., 19, 377-389

Wilkinson, J. (2010). Economic and agro food studies in Brazil: combining social networks, convention and social movements approaches. Econ. Soc., 11(2), 23-32.

Zepeda, J. A., Vergara, M., Gómez, A., Espinosa, M., Espinoza, A. \& López, A. (2019). Beneficios de las etiquetas en pesquerías en vías de desarrollo: capítulo México. En G. Ponce \& F. González-Laxe (Eds.), Gobernanza Pesquera (pp.163-180). México. Instituto Politécnico Nacional. 
